\title{
Experiencia con paratiroidectomía quirúrgica en pacientes con enfermedad renal crónica e hiperparatiroidismo terciario
}

\author{
Experience with surgical parathyroidectomy in \\ patients with chronic kidney disease and tertiary \\ hyperparathyroidism
}

\author{
Valentina Sanint, César Augusto Restrepo, Andrés Ignacio Chala • \\ Manizales (Colombia)
}

\section{Resumen}

Introducción: el hiperparatiroidismo terciario es la consecuencia final de las alteraciones del metabolismo calcio-fósforo en pacientes con enfermedad renal crónica. Aquellos pacientes que no logran controlarlo con el tratamiento médico, requieren paratiroidectomía.

Objetivo: describir la evolución clínica y paraclínica en pacientes con enfermedad renal crónica y diagnóstico de hiperparatiroidismo terciario, que fueron llevados a paratiroidectomía.

Metodología: estudio prospectivo, que incluyó pacientes con hiperparatiroidismo terciario llevados a paratiroidectomía entre los años 2006 y 2015. Se realizaron estudios bioquímicos pre y post quirúrgicos y se evaluó la presencia y progresión de síntomas.

Resultados: se incluyeron 32 pacientes, $68.8 \%$ mujeres, con media de edad de 46.2 años. Se identificaron diferencias estadísticamente significativas en el cambio en los valores de calcio, fósforo y PTH pre y post quirúrgicos. El 81.5\% de los pacientes reportaron presencia de síntomas. El 34.6, 26.9, y $23 \%$ presentaron mejoría total de dolor óseo, dolor articular y prurito, respectivamente. El $28 \%$ presentaron hipocalcemia sintomática post operatoria. No hubo casos de muerte durante el seguimiento.

Conclusiones: la paratiroidectomía se constituye en un alternativa segura y confiable para los pacientes con hiperparatiroidismo terciario, mejorando significativamente los síntomas y las alteraciones en el metabolismo óseo y mineral. Vigilar los niveles de calcio en el post operatorio es importante para evitar las complicaciones del síndrome de hueso hambriento. (Acta Med Colomb 2018; 43: 136-141).

Palabras clave: insuficiencia renal crónica, hiperparatiroidismo, paratiroidectomía, osteodistrofia renal, diálisis, trasplante de riñón.

\footnotetext{
Abstract

Introduction: tertiary hyperparathyroidism is the final consequence of alterations in calciumphosphorus metabolism in patients with chronic kidney disease. Those patients who fail to control it with medical treatment require parathyroidectomy.

Objective: go describe the clinical and paraclinical evolution in patients with chronic kidney disease and diagnosis of tertiary hyperparathyroidism, who underwent parathyroidectomy.

Methodology: prospective study, which included patients with tertiary hyperparathyroidism taken to parathyroidectomy between 2006 and 2015. Pre and post-surgical biochemical studies were performed and the presence and progression of symptoms was evaluated.

Results: 32 patients were included. $68.8(\%)$ were women with an average age of 46.2 years. Statistically significant differences were identified in the change in calcium, phosphorus and PTH values before and after surgery. $81.5 \%$ of patients reported the presence of symptoms. 34.6, 26.9, and $23 \%$ presented total improvement of bone pain, joint pain and pruritus, respectively. $28 \%$ presented postoperative symptomatic hypocalcemia. There were no deaths during follow-up.
}

Dra. Valentina Sanint Castro: Residente IV Año Medicina Interna y Geriatría. Universidad de Caldas; Dr. César Augusto Restrepo Valencia: Internista Nefrólogo. Profesor Asociado Universidad de Caldas y Universidad de Manizales; Dr. Andrés Ignacio Chala Galindo: Médico Cirujano de Cabeza y Cuello. Profesor Asociado Universidad de Caldas. Manizales (Colombia). Correspondencia. Dr. César Augusto Restrepo Valencia. Manizales (Colombia).

E-mail: caugustorv@une.net.co Recibido: 21/V/2017 Aceptado: 9/V/2018 
Conclusions: parathyroidectomy is a safe and reliable alternative for patients with tertiary hyperparathyroidism, significantly improving symptoms and alterations in bone and mineral metabolism. Monitoring calcium levels in the postoperative period is important to avoid the complications of the hungry bone syndrome. (Acta Med Colomb 2018; 43: 136-141).

Keywords: chronic renal failure, hyperparathyroidism, parathyroidectomy, renal osteodystrophy, dialysis, kidney transplant.

\section{Introducción}

El hiperparatiroidismo terciario es un problema frecuente en los pacientes con enfermedad renal crónica (ERC) en sus estadios más avanzados, y trasplantados renales, principalmente en aquellos quienes previamente estuvieron en terapia dialítica prolongada (1). Se caracteriza por funcionamiento autónomo de las glándulas paratiroideas, con aumento en la síntesis y secreción de parathormona (PTH) a pesar de la prescripción de diversas terapias médicas. Se asocia a alteraciones del metabolismo del fósforo, calcio, vitamina $\mathrm{D}, \mathrm{y}$ a trastornos minerales y óseos, que se van a ver manifestados por múltiples síntomas (2). Aquellos pacientes que no responden al manejo farmacológico requieren resección quirúrgica de las glándulas paratiroides (3). Se desconoce con exactitud cuál es el perfil de pacientes que responden al manejo quirúrgico, cuál es el grado de mejoría de síntomas y de perfil bioquímico, y cuál es la evolución a largo plazo tras el procedimiento (4). El objetivo de nuestro estudio es describir la evolución clínica y paraclínica en pacientes con enfermedad renal estadío $\mathrm{V}$ y pacientes trasplantados renales, con diagnóstico de hiperparatiroidismo terciario, que fueron llevados a paratiroidectomía total con autotrasplante.

\section{Material y métodos}

Estudio de tipo descriptivo prospectivo. Se captaron pacientes mayores de 18 años con diagnóstico de enfermedad renal crónica estadío $\mathrm{V}$ en terapia de reemplazo renal o pacientes trasplantados renales, que tuvieran diagnóstico de hiperparatiroidismo terciario y que fueron llevados a paratiroidectomía total con autotrasplante, valorados en la consulta de nefrología entre los años 2006 y 2015. Se excluyeron los pacientes con datos insuficientes para su análisis. Se tomaron los datos clínicos y paraclínicos de interés.

Se definió hiperparatiroidismo terciario con indicación quirúrgica a aquellos pacientes que cumplieran con las siguientes características: Elevación persistente y progresiva de la PTH, con niveles superiores a $800 \mathrm{pg} / \mathrm{mL}$, a pesar de manejo médico óptimo por más de seis meses con calcitriol o agonistas selectivos del receptor de la vitamina D (ningún paciente fue tratado con cinacalcet). Hallazgos ecográficos, en los que se encontrara una glándula paratiroides con volumen $\geq 1000 \mathrm{~mm}^{3}$, o 2 o más glándulas paratiroides con volúmenes superiores a $500 \mathrm{~mm}^{3}$. Pacientes post trasplante renal, en quienes después de seis meses se identificó persistente ó nueva elevación de la PTH, con ausencia de respuesta a terapia clásica y hallazgos ecográficos compatibles con adenomas paratiroideos. Se registró las modificaciones en las variables bioquímicas calcio, fósforo, producto calcio $\mathrm{x}$ fósforo, fosfatasa alcalina, PTH antes de la cirugía y tres, seis, nueve y 12 meses postoperatorios, al igual que los síntomas compatibles con hiperparatiroidismo,

El análisis de la información se realizó usando el programa SPSS, versión 15 licenciado para la Universidad de Caldas. Se utilizaron medidas de tendencia central y de dispersión con las variables cuantitativas y distribución de frecuencias absolutas y relativas con las variables cualitativas. Para cada uno de los reportes de laboratorio se calculó ttest para muestras relacionadas y Wilcoxon. Se consideró un valor de p menor a 0.05 como estadísticamente significativo.

El proyecto fue aprobado por el comité de ética de la Universidad de Caldas y comités de investigaciones y ética de RTS Colombia.

\section{Resultados}

Durante el periodo de estudio comprendido entre enero de 2006 y diciembre de 2015, se identificaron 32 pacientes que cumplían los criterios de inclusión para su evaluación. El 90.6\% (29 pacientes) se encontraban en terapia dialítica y $9.3 \%$ (tres pacientes) eran trasplantados renales. De los pacientes en diálisis la distribución entre sus variedades diálisis peritoneal y hemodiálisis fue similar, con 51\% (15 pacientes) y $49 \%$ (14 pacientes) respectivamente. La media de tiempo desde el inicio de la diálisis hasta el momento de la cirugía fue de 7.5 años, y $37 \%$ (10 pacientes) de los pacientes tuvieron tiempos de diálisis hasta la cirugía $\geq 10$ años (Tabla 1).

La principal causa de enfermedad renal crónica en nuestros pacientes fue nefropatía hipertensiva en $28 \%$ seguida

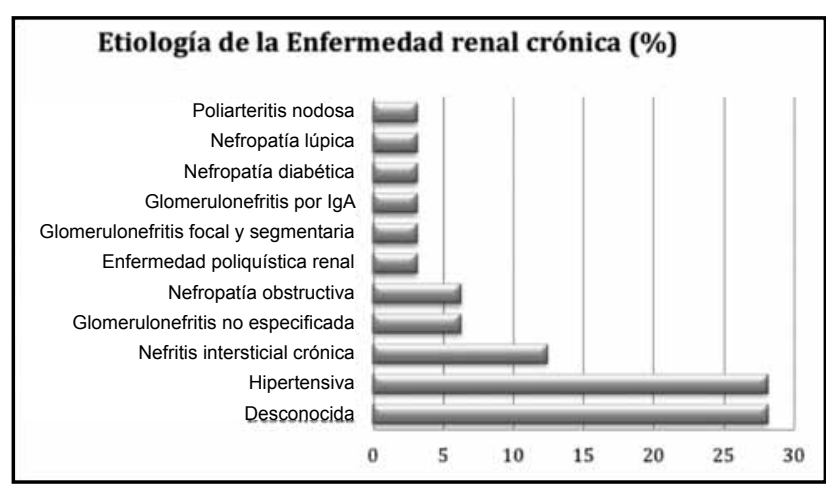

Figura 1. Etiología de la enfermedad renal crónica identificada en los pacientes intervenidos 


\begin{tabular}{|c|c|c|c|c|c|}
\hline Variable & $\begin{array}{c}\text { Frecuencia } \% \\
(\mathbf{n}: 32)\end{array}$ & Media & $\begin{array}{c}\text { Desviación } \\
\text { estándar }\end{array}$ & Mínimo & Máximo \\
\hline Edad (años) & - & 46,2 & 13,8 & 18 & 76 \\
\hline Sexo (mujer) & $68.8(22)$ & - & - & - & - \\
\hline Pacientes en diálisis & $90.6(29)$ & & & & \\
\hline Diálisis peritoneal & $51(15)$ & & & & \\
\hline Hemodiálisis & $49(14)$ & & & & \\
\hline Pacientes trasplantados & $9.3(3)$ & & & & \\
\hline Tiempo de diálisis & - & 7,5 & 3,5 & 2 & 14 \\
\hline Diálisis mayor a 10 años & $37(10)$ & & & & \\
\hline $\begin{array}{l}\text { - Presencia de síntomas } \\
\text { - Dolor articular } \\
\text { - Dolor óseo } \\
\text { - Prurito } \\
\text { - Piel seca }\end{array}$ & $\begin{array}{l}81.5(26) \\
81.5(26) \\
81.5(26) \\
81.5(26) \\
75(25)\end{array}$ & & & & \\
\hline $\begin{array}{l}\text { Complicaciones a un año de seguimiento } \\
\text { - Hipocalcemia sintomática } \\
\text { - Peritonitis } \\
\text { - Muerte }\end{array}$ & $\begin{array}{l}32(11) \\
28(10) \\
3(1) \\
0(0)\end{array}$ & & & & \\
\hline
\end{tabular}

de nefritis intersticial crónica (12.4\%), 28\% de los casos fueron de etiología desconocida (Figura 1).

Se realizó comparación de los valores bioquímicos un mes antes del procedimiento quirúrgico, con los valores obtenidos a los tres, seis, nueve y 12 meses post operatorios. Encontramos diferencias estadísticamente significativas en los valores de calcio medidos un mes antes del procedimiento quirúrgico y los meses tres y nueve post operatorios ( $\mathrm{p}=$ 0.038 y 0.022 respectivamente) (Figura 2).

Hubo además diferencias estadísticamente significativas entre el valor de fósforo un mes antes de la cirugía y los meses tres $(\mathrm{p}=0.019)$, seis $(\mathrm{p}=0.008)$, nueve $(\mathrm{p}=0.012)$ y $12(\mathrm{p}=0.008)$ post operatorios, al igual que en los valores del producto calcio $x$ fósforo $(\mathrm{p}=0.001,0.009-0.003-0.001$ respectivamente) (Figuras 3 y 4 ).

En los valores de fosfatasa alcalina se observa un ascenso progresivo desde tres meses antes de la cirugía hasta el

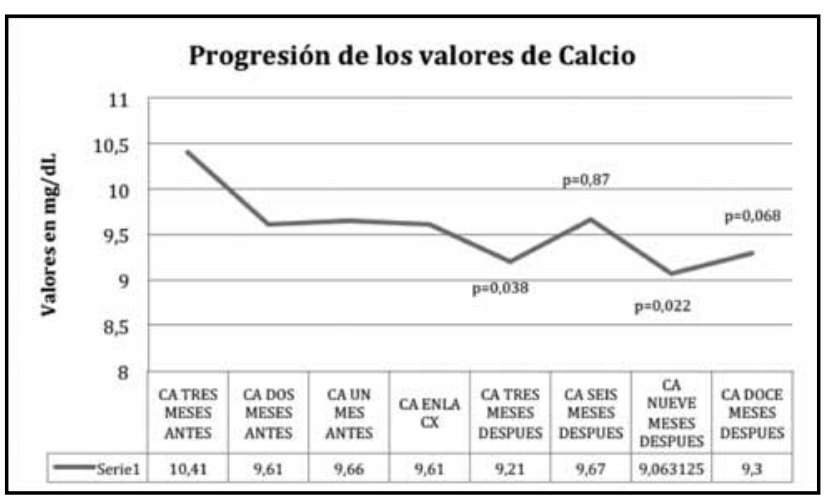

Figura 2. Cambio en los valores de calcio sérico a lo largo del periodo de observación en $m g / d L$. momento del procedimiento, y posterior a la cirugía con descenso progresivo hasta el año de seguimiento. Hubo diferencias estadísticamente significativas entre el valor un mes antes de la cirugía y los valores a los nueve $(\mathrm{p}=0.043)$ y $12(\mathrm{p}=0.011)$ meses post operatorios (Figura 5).

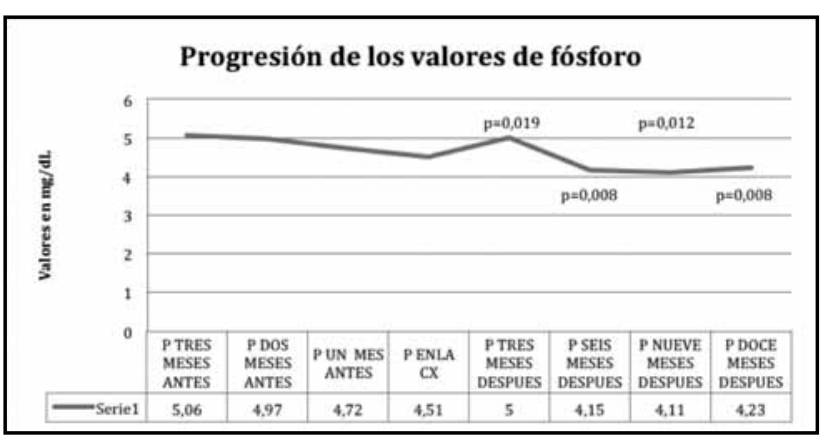

Figura 3. Evolución en los valores de fósforo sérico a lo largo del periodo de observación en $m g / d L$.

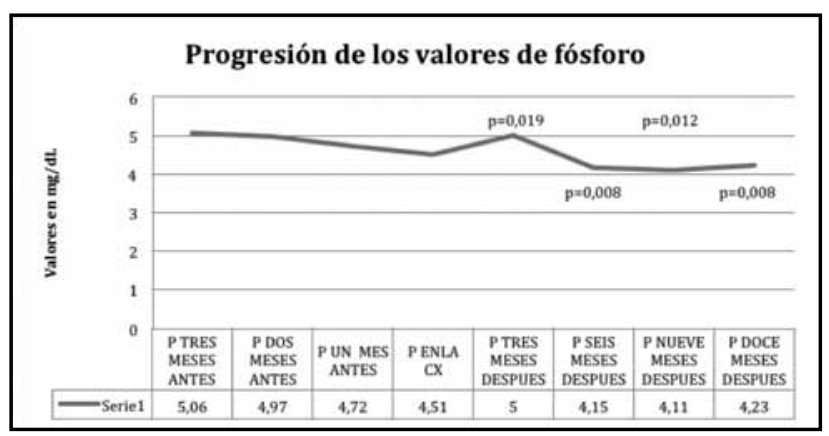

Figura 4. Cambio en los valores del producto calcio x fósforo a lo largo del periodo de observación en valores absolutos. 


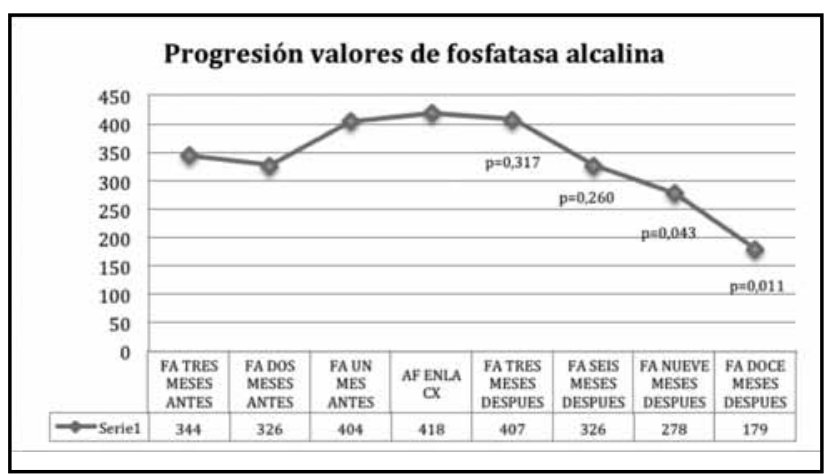

Figura 5. Progresión en los valores de fosfatasa alcalina a lo largo del periodo de observación en $\mathrm{mg} / \mathrm{dL}$.

El valor medio de PTH tres meses antes de la cirugía fue de 1406 pg/dL, con un valor máximo de 2500 pg/dL. La media de PTH a los 12 meses de seguimiento fue de $249 \mathrm{pg} / \mathrm{dL}$, con una mínima de $3 \mathrm{pg} / \mathrm{dL}$ y una máxima de $1016 \mathrm{pg} / \mathrm{dL}$. Al comparar los valores de PTH un mes antes de la cirugía con los tres, seis, nueve y 12 meses postquirúrgicos, hubo diferencias estadísticamente significativas con todos ellos, con valores de $\mathrm{p}<0.0001$ en todos los casos. El descenso más marcado se observa a los tres meses luego del procedimiento, manteniéndose estable durante los 12 meses siguientes (Figura 6).

\section{Variables clínicas}

Dentro de la población evaluada, se encontró que el $81.5 \%$ de los pacientes reportaron la presencia de síntomas. De los pacientes que reportaron síntomas, el $100 \%$ de ellos presentaron dolor articular, óseo y prurito y $96 \%$ presentaron piel seca.

De los pacientes que se presentaron con dolor articular (26 pacientes), el $26.9 \%$ presentaron mejoría completa del mismo, $38.4 \%$ tuvieron mejoría parcial y $34.6 \%$ no presentaron ninguna mejoría. En cuanto al dolor óseo (26 pacientes), $34.6 \%$ presentaron mejoría total, $30.7 \%$ mejoría parcial y $34.6 \%$ no tuvieron mejoría. De los pacientes con piel seca (25 pacientes), $24 \%$ manifestaron mejoría total, $48 \%$ presentaron mejoría parcial y $28 \%$ no presentaron mejoría. De los pacientes con prurito (26 pacientes), $23 \%$ presentaron mejoría total, $30.7 \%$ presentaron mejoría parcial y $46.1 \%$ ninguna mejoría.

El $68 \%$ de los pacientes no presentaron complicaciones asociada a la cirugía, $28 \%$ presentaron hipocalcemia sintomática y $3 \%$ (un solo paciente que se encontraba en diálisis peritoneal) presentó peritonitis intrahospitalaria. Durante el año de seguimiento no se presentó ningún caso de muerte.

\section{Discusión}

La generación del hiperparatiroidismo en la ERC es un proceso complicado y extenso que ha sido resumido en forma detallada recientemente (5). Hiperparatiroidismo terciario en pacientes con ERC se define como la incapacidad en obtener niveles satisfactorios de PTH a pesar de la terapia médica aplicada. Esta última ha evolucionado

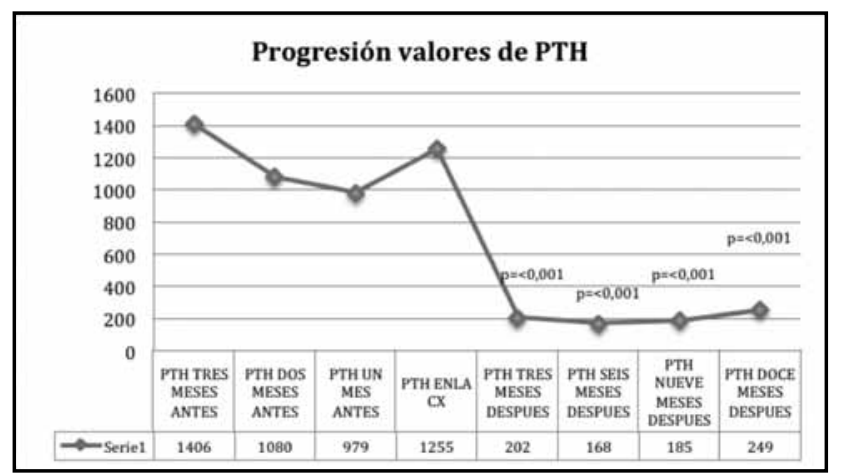

Figura 6. Evolución en los valores de PTH a lo largo del periodo de observación en pg/dL.

desde la utilización de calcio en sus diversas presentación (carbonato, acetato), vitamina D activa (calcitriol), agonistas selectivos del receptor de la vitamina D (paricalcitol), hasta los más recientes calcimiméticos de uso oral (cinacalcet) o parenteral (etecalcetide) (6). Una característica primordial para su diagnóstico es la presencia de hiperplasia nodular de la glándula paratiroides, la cual le confiere un carácter autónomo, con reducción muy significativa en la expresión de receptores al calcio y a la vitamina $\mathrm{D}$ activa (calcitriol) (7 - 9). En diversos estudios que incluyen variables bioquímicas, imagenológicas y análisis histopatológico de glándulas paratiroides, se ha demostrado muy buena correlación entre la refractariedad a la terapia médica y el volumen de las glándulas paratiroides detectado en la ecografía de cuello $(10,11)$, siendo una recomendación la práctica de estudios imagenológicos (ecografía de cuello o gamagrafía de paratiroides) ante la ausencia de respuesta terapéutica (12).

El hiperparatiroidismo es una complicación de la ERC que afecta notablemente la calidad de vida, incrementando la mortalidad cardiovascular y el riesgo de facturas $(13,14)$.

El tratamiento del hiperparatiroidismo terciario puede llevarse a cabo con inyección percutánea de las glándulas paratiroides guiada por ecografía bien sea con etanol o paricalcitol, basándose su elección en el numero de glándulas paratiroides y el riesgo quirúrgico del paciente $(15,16)$.

La paratiroidectomia se recomienda en casos de persistente hiperfosfatemia o hipercalcemia a pesar de terapia médica, evidencia de calcificaciones extraóseas (de tejidos blandos), calcifilaxis, prurito severo, niveles de PTH persistentemente elevados (mayores a 800 pg $(\mathrm{mL})$, y glándulas paratiroideas con volumen superior a $1000 \mathrm{~mm}^{3}(17,18)$.

Hay diversas técnicas para la paratiroidectomía: parcial o subtotal y total, esta última con o sin autotrasplantante, dando lugar a diversos resultados terapéuticos $(20,21)$.

En cuanto a su beneficio en varios estudios se demuestra gran mejoría en el metabolismo fosfo-calcio, con reducción significativa en los valores de calcio, fósforo y PTH $(22,23)$.

Hay reportes también de mejoría en los resultados cardiovasculares y sobrevida principalmente en pacientes en hemodiálisis crónica (24 - 26). 
La calidad de vida de los pacientes también mejora notablemente al ser evaluada por diversos indicadores $(27,28)$, y siendo analizada con base en búsquedas de la literatura sistémica (29).

Los síntomas atribuibles al hiperparatiroidismo son artralgias, dolores óseos difusos, mialgias, debilidad muscular y prurito. En varios estudios se describe reducción muy significativa en los síntomas posterior a la paratiroidectomía $(30,31)$.

Las complicaciones del procedimiento quirúrgico incluyen hipocalcemia (síndrome del hueso hambriento), aumento en las visitas hospitalarias y alteraciones cardiovasculares entre las que resaltan arritmias cardiacas y eventos cerebrovasculares (32).

Las tasas de mortalidad se han informado en valores cercanos a $2 \%$ a los 30 días después del procedimiento (32).

La implementación de la utilización de vitamina $\mathrm{D}$ en cualquiera de sus formas, y lograr niveles de calcio sérico normales días antes de la cirugía reduce la aparición de hipocalcemia en el post operatorio $(33,34)$.

En pacientes trasplantados renales en un estudio altos niveles de PTH pre trasplante se asociaron en el post quirúrgico con trombosis microvascular y retraso en la función del injerto (35), pero en otro estudio de carácter retrospectivo no se replicaron esos hallazgos (36).

La paratiroidectomía previa al trasplante en pacientes con hiperparatiroidismo reduce la falla del injerto en 12 años de seguimiento, pero no afecta el retraso en la función del injerto, ni las complicaciones de todas las causas ni desequilibrios del calcio (37). De todos modos la paratiroidectomía en pacientes con hiperparatiroidismo persistente post trasplante constituye una alternativa segura y efectiva como se sugiere en una publicación en la cual se informa de los resultados de 15 pacientes sometidos al procedimiento (38).

En nuestro estudio que se extendió a nueve años encontramos durante el seguimiento pre y post quirúrgico una reducción muy significativa en los valores de calcio, fósforo, producto calcio x fósforo y PTH, subrayándose la efectividad de la paratiroidectomía en reducir la PTH. El $81.5 \%$ de nuestros pacientes reportaron presencia de síntomas relacionados con hiperparatiroidismo, sugiriendo que la paratiroidectomía se sigue de alivio inmediato de los síntomas clínicos. En relación a las complicaciones $31 \%$ de los pacientes las presentaron, el $28 \%$ con hipocalcemia sintomática. Durante el año de seguimiento no se presentó ningún caso de mortalidad.

El seguimiento de nuestros pacientes fue a 12 meses, y no es posible entonces evaluar efectos del procedimiento quirúrgico a largo plazo y tampoco efectos sobre supervivencia,

Se resalta de nuestro estudio que es el primero de este tipo realizado hasta la fecha en Colombia. La evaluación y tratamiento de los pacientes fue estandarizada, al igual que los estudios de laboratorio. Todos los pacientes fueron intervenidos por el mismo cirujano de cabeza y cuello, empleando igual técnica quirúrgica en todos los casos.
Dentro de las limitaciones de nuestro estudio se encuentra que la evaluación de la sintomatología fue de carácter subjetivo que limita su comparación con otros estudios similares. Se incluyeron pacientes de una única unidad renal de la ciudad de Manizales, lo que puede limitar la generalización de los resultados.

Podemos concluir en nuestro estudio que la paratiroidectomía en manos de cirujanos experimentados es un procedimiento seguro y efectivo para el tratamiento del hiperparatiroidismo en pacientes con ERC refractarios a terapia médica.

\section{Agradecimientos}

Luz Yaneth Becerra, Asesora epidemiológica. Médica cirujana Universidad de Caldas. Especialista en Epidemiología.

\section{Referencias}

1. Damasiewicz MJ, Ebeling PR. Management of mineral and bone disorders in renal transplant recipients. Nephrology (Carlton). 2017; 22 Suppl 2:65-69.

2. Hawley CM, Holt SG. Parathyroid hormone targets in chronic kidney disease and managing severe hyperparathyroidism. Nephrology (Carlton). 2017; 22, Suppl. $2: 47-50$.

3. Dulfer RR, Franssen GJH, Hesselink DA, Hoorn EJ, van Eijck CHJ, van Ginhoven TM. Systematic review of surgical and medical treatment for tertiary hyperparathyroidism. Br J Surg. 2017;104(7):804-813.

4. Dewberry LC, Tata S, Graves S, Weber CJ, Sharma J. Predictors of tertiary hyperparathyroidism: Who will benefit from parathyroidectomy? Surgery. 2014;156(6): 1631-1636

5. Restrepo C A, Giraldo G C. Metabolismo fosfo-calcico en la enfermedad renal crónica. In: Musso CG, Greloni G, Imperiali N, Rosa Diez G, editores. Enfermedad renal crónica. Buenos aires. Delhospitalediciones. 2017. (In press).

6. Block GA, Bushinsky DA, Cheng S, Cunningham J, Dehmel B, Drueke TB et al. Effect of Etelcalcetide vs Cinacalcet on Serum Parathyroid Hormone in Patients Receiving Hemodialysis With Secondary Hyperparathyroidism A Randomized Clinical Trial. JAMA 2017; 317(2):156-164.

7. Tominaga Y, Kohara S, Namii Y, Nagasaka T, Haba T, Uchida K et al. Clonal analysis of nodular parathyroid hyperplasia in renal hyperparathyroidism. World J Surg 1996;20(7):744-750.

8. Gogusev J, Duchambon P, Hory B, Giovannini M, Goureau Y, Sarfati E et al. Depressed expression of calcium receptor in parathyroid gland tissue of patients with hyperparathyroidism. Kidney Int 1997;51(1):328-336.

9. Fukuda N, Tanaka H, Tominaga Y, Fukagawa M, Kurokawa K, Seiko Y. Decreased 1,25-dihydroxyvitamin D3 receptor density is associated with a more severe form of parathyroid hyperplasia in chronic uremic patients. J Clin Invest 1993;92(3):1436-1443.

10. Matsuoka S, Tominaga Y, Sato T, Uno N, Hiramitu T, Goyo N et al. Relationship between the dimension of parathyroid glands estimated by ultrasonography and the hyperplastic pattern in patients with renal hyperparathyroidism. Ther Apher Dial 2008;12(5): 391-395

11. Indridason O, Heath III H, Khosla S, Yohay DA, Quarles D. Non-suppressible parathyroid hormone secretion is related to gland size in uremic secondary hyperparathyroidism. Kidney Int 1996; 50(5): 1663-1671.

12. Restrepo C A, Santacruz D, Castillo C E, Chacon J A. Detección de hiperplasia de paratiroides por ultrasonografía y correlación con signos clínicos y de laboratorio en pacientes con enfermedad renal crónica. Rev Colomb Radiol 2011 22(4): 3341-3347.

13. Davies EW, Matza LS, Worth G, Feeny DH, Kostelec J, Soroka SD et al. Impact of major clinical events on utilities in the context of secondary hyperparathyroidism (SHPT) and chronic kidney disease (CKD) treated with dialysis. Value Health 2013; 16(7): A634

14. Danese MD, Kim J, Doan QV, Dylan M, Griffith R, Chertow GM. PTH and the risks for hip, vertebral, and pelvic fractures among patients on dialysis. Am J Kidney Dis 2006;47 (1): 149-156.

15. Restrepo C A, Castillo C E, Sanz M F, Velez C. La eficacia del etanol intraglandular en hiperparatiroidismo secundario se limita a una sola glándula hiperplásica. Acta Med Colomb 2013; 38(2): 61-67.

16. Restrepo CA, Castillo C E, Sanz M F. Aplicación seriada de paricalcitol intrano- 
dular ¿es eficaz en el tratamiento del hiperparatiroidismo secundario refractario?. Acta Med Colomb 2015; 40 (2): 125-131.

17. Guideline working Group, Japanese society for dialysis therapy. Clinical practice guideline for the management of secondary hyperparathyroidism in chronic dialysis patients. Ther Apher Dial 2008; 12(6): 514-525.

18. Kidney Disease: Improving Global Outcomes (KDIGO) CKD- MBD Work Group. KDIGO clinical practice guideline for the diagnosis, evaluation, prevention, and treatment of Chronic Kidney Disease-Mineral and Bone Disorder (CKD-MBD). Kidney Int 2009; Suppl 113: S1-S130.

19. Hamouda M, Ben Dhia N, Alousi G Gorsane I, Skhiri H, Frih A, et al. Surgical treatment of secondary hyperparathyroidism in patients with chronic renal failure. Nephrol Ther. 2011;7(2):105-110.

20. Iwamoto, Sato N, Nishida M, Hashimoto T, Kobayashi H, Yamasaki S, et al. Total parathyroidectomy improves survival of hemodialysis patients with secondary hyperparathyroidism. J Nephrol 2012; 25(5):755-763.

21. Shih ML, Duh QY, Hsieh CB, Lin SH, Wu HS, Chu PL, Chen TY, Yu JC. Total parathyroidectomy without autotransplantation for secondary hyperparathyroidism. World J Surg. 2009;33(2):248-54.

22. Conzo G, Perna AF, Sinisi AA, Palazzo A, Stanzione F, Della Pietra C, Livrea A. Total parathyroidectomy without autotransplantation in the surgical treatment of secondary hyperparathyroidism of chronic kidney disease. J Endocrinol Invest. 2012;35(1):8-13.

23. Fotheringham J, Balasubramanian SP, Harrison B, Wilkie M. Post-parathyroidectomy parathyroid hormone levels: the impact on patient survival - a single-centre study in a stage 5 chronic kidney disease population. Nephron Clin Pract. 2011;119(2): c113-20.

24. Conzo G, Perna AF, Savica V, Palazzo A, Pietra CD, Ingrosso D et al. Impact of parathyroidectomy on cardiovascular outcomes and survival in chronic hemodialysis patients with secondary hyperparathyroidism. A retrospective study of 50 cases prior to the calcimimetics era. BMC Surg 2013; 13(suppl2) : S4.

25. Iwamoto N, Sato N, Nishida M, Hashimoto T, Kobayashi H, Yamasaki S, et al. Total parathyroidectomy improves survival of hemodialysis patients with secondary hyperparathyroidism. J Nephrol. 2012;25(5):755-763.

26. Komaba H, Taniguchi M, Wada A, Iseki K, Tsubakihara Y, Fukagawa M. Parathyroidectomy and survival among Japanese hemodialysis patients with secondary hyperparathyroidism. Kidney Int . 2015;88(2):350-359.

27. Cheng SP, Lee JJ, Liu TP, Yang TL, Chen HH, Wu CJ et al. Parathyroidectomy improves symptomatology and quality of life in patients with secondary hyperparathyroidism. Surgery 2014;155(2): 320-328.
28. Dulfer R, Geilvoet W, Morks A, van Lieshout EM, Smit CP, Nieveen van Dijkum EJ, et al. Impact of parathyroidectomy for primary hyperparathyroidism on quality of life: a case-control study using Short Form Health Survey 36. Head Neck 2016; 38(8): 1213-1220.

29.van der Plas WY, Dulfer RR, Engelsman AF, Vogt L, de Borst MH, van Ginhoven TM et al; Dutch Hyperparathryoid Study Group(DHSG). Effect of parathyroidectomy and cinacalcet on quality of life in patients with end-stage renal disease-related hyperparathyroidism: a systemic review. Nephrol Dial Transplant. 2017 Apr 10. doi: 10.1093/ndt/gfx044. [Epub ahead of print]).

30. Drakopoulos S, Koukoulaki M, Apostolou T, Pistolas D, Balaska K, Gavriil S, et al. Total parathyroidectomy without autotransplantation in dialysis patients and renal transplant recipients, long-term follow-up evaluation. Am J Surg. 2009;198(2): 178-183.

31. Cheng SP, Lee JJ, Liu TP, Yang TL, Chen HH, Wu CJ, et al. Parathyroidectomy improves symptomatology and quality of life in patients with secondary hyperparathyroidism. Surgery. 2014;155(2):320-328.

32. Ishani A, Liu J, Wetmore JB et al. Clinical outcomes after parathyroidectomy in a nationwide cohort of patients on hemodialysis. Clin J Am Soc Nephrol 2015; 10: $90-97$.

33. Goonewardene SS, Ready A. Parathyroidectomies: Pre and Post Op Usage of Calcium Supplementation and Effect on Calcium Levels. Arch Renal Dis Manag 2016; 2(1): 005-007.

34. Latus J, Roesel M, Fritz P, Braun N, Ulmer C, Steurer W, Biegger D, Alscher MD, Kimmel M. Incidence of and risk factors for hungry bone syndrome in 84 patients with secondary hyperparathyroidism. Int J Nephrol Renovasc Dis 2013; 8(6): $131-137$.

35. Roodnat JI, van Gurp EA, Mulder PG, van Gelder T, de Rijke YB, de Herder WW, et al. High pretransplant parathyroid hormone levels increase the risk for graft failure after renal transplantation. Transplantation. 2006 15;82(3):362-367.

36. Molnar MZ, Kovesdy CP, Mucsi I, Salusky IB, Kalantar-Zadeh K. Association of pre-kidney transplant markers of mineral and bone disorder with post-transplant outcomes. Clin J Am Soc Nephrol. 2012 ;7(11):1859-1871.

37. Callender GG, Malinowski J, Javid M, Zhang Y, Huang H, Quinn CE, et al. Parathyroidectomy prior to kidney transplant decreases graft failure. Surgery. 2017;161(1):44-50.

38. Meng C, Martins P, Frazão J, Pestana M. Parathyroidectomy in Persistent Post-transplantation Hyperparathyroidism - Single-center Experience. Transplant Proc. 2017;49(4):795-798. 